Research Article

\title{
Research on Green Habitat Design Based on Field Wireless Sensing Hazardous Substance Detection
}

\author{
Wei Xiong $\mathbb{D I}^{1}$ and Liangquan $\mathrm{Hu} \mathbb{D}^{2}$ \\ ${ }^{1}$ School of Architecture, Southeast University, Jiangsu, Nanjing 210018, China \\ ${ }^{2}$ Architectural Design \& Research Institute, Hefei Construction Engineering Group Co., Ltd, Hefei, Anhui 230088, China \\ Correspondence should be addressed to Wei Xiong; 230139314@seu.edu.cn
}

Received 18 September 2021; Revised 9 October 2021; Accepted 11 October 2021; Published 28 October 2021

Academic Editor: Guolong Shi

Copyright (c) 2021 Wei Xiong and Liangquan Hu. This is an open access article distributed under the Creative Commons Attribution License, which permits unrestricted use, distribution, and reproduction in any medium, provided the original work is properly cited.

\begin{abstract}
Field wireless sensor network is the current global engineering field research hotspot for structural health monitoring wireless sensor network that is one of the important branches to real-time monitoring of the safety status of the upper wood engineering structure to avoid the occurrence of many safety accidents caused by major structural and equipment damage and to guide the maintenance of major structures; the establishment of a wireless sensor network system is one of the current research priorities. This paper researches and designs a wireless sensor network system level scheme for structural health monitoring that is divided into two parts based on the hardware platform design and software development based on the system that focuses on the time synchronization protocol and synchronous acquisition method featuring synchronous acquisition start time scheme, time separation method, and flexible optimization model of time information. The method applies to high-frequency acquisition to guarantee the time of sampling points in structural environmental measurement. The accuracy of the information and the reliability of the field diagnosis, for the detection of harmful substances, as well as leading to the construction of green habitat environment have a qualitative leap, for the design of green habitat environment that has enough progress.
\end{abstract}

\section{Introduction}

Wireless sensor networks are usually energy limited and susceptible to environmental interference, in order to improve the energy efficiency of wireless sensor networks and guarantee data reliability, and energy-efficient data fusion algorithm (EEDFA) based on a split cluster wireless sensor network is proposed; EEDFA operates in a network with a split cluster structure; the data acquisition and transmission process are divided into multiple cycles; the sensors collect data in multiple time slots within the cycle to form data vector each cycle the sensor downscales the collected data vector to reduce the transmission load, while considering the environmental interference using interval type-two fuzzy system to generate data reliability factor to guarantee the reliability of the data each cluster head receives the data from the member nodes for distributed processing and removes the redundant data using wood similarity function and sends the fused data to the next hop cluster head or base station simulation results show that compared to PFF REDA. SCDRE EEDFA has lower bandwidth usage and energy consumption and also has higher data accuracy [1].

Wireless sensor networks (WSNs) are composed of a large number of miniature sensor nodes that combine sensing capability, computing capability, and short-range communication capability deployed in a monitoring area, whose nodes are generally powered by batteries that carry limited energy, and the energy is usually difficult to be replenished. To extend the network life cycle, it is necessary to reduce the network energy consumption in WSNs [2]; to monitor events more accurately, sensor nodes are usually deployed densely, which may lead to the high spatial correlation between data collected by multiple neighboring sensors, and at the same time, there are temporal correlations between data continuously collected by individual sensor nodes, and these correlations make a large amount of 
redundant data in the network. In addition, sensor nodes may sense unreliable data due to problems such as environmental noise and node failure. Data fusion is a data processing process that uses computer-related techniques to analyze and organize the data collected by sensors in a certain way, which can reduce redundant or unreliable data and improve energy efficiency.

In recent years, many scholars have proposed some efficient data fusion algorithms in the field of data fusion. Some variable weight-based data fusion algorithms have been proposed in the literature. These variable weight fusion algorithms effectively reduce the transmission load by weighting the data on average. However, it greatly reduces the integrity of the data, which may lead to poor reliability of the final judgment events. In the literature, the authors proposed a prefix frequency filtering- (PFF-) based data fusion algorithm, which achieves the fusion of sensory data in two steps: In the first step, the data is initially weighted down and sorted at the sensor nodes; in the second step, the PFF and Jaccard similarity are used in the aggregator to the data between the nodes are fused. The method can quickly remove redundant data, but after the data are sorted, the order is changed, and the time information is lost. The REDA algorithm proposed in the literature uses a splitcluster structure to fuse the data at the common nodes and cluster heads, respectively, and removes redundant data with pattern codes, which improves the bandwidth utilization of the data, but the pattern codes are not effective in identifying similar data SCDRE algorithm that performs similarity analysis on data collected between different sensor nodes using Pearson correlation coefficient to remove similar data; however, using correlation coefficient alone is not a good measure of data similarity. In addition, all these algorithms assume that the collected data is correct without judging the credibility of the data source, not only the accuracy of the data is not guaranteed but also increases the unnecessary communication overhead [3]. The first-generation sensor network of traditional sensors has a transmission function for point-to-point signals, using a two-wire 4-20 mA current or $1 \sim 5 \mathrm{~V}$ voltage standard to realize one-way transmission of information. The second-generation sensor network is a measurement and control network composed of intelligent sensors and on-site control stations. Use analog current or voltage signal to realize sensor signal transmission. The third-generation sensor network is based on the Fieldbus intelligent sensor network. Fieldbus is a fully digital, open two-way communication network connecting intelligent field devices and control rooms.

\section{Related Work}

The global research on WSNs began with research projects supported by the literature. It has been widely studied by governments, schools, and related research institutions since the 1990s. The literature began researching Smart Sensor Network Communications (SSNC) [4], which uses "throwaway" sensors to collect battlefield information and plans to build a smart network that can interconnect with robots used in future combat systems. The literature proposes the concept of smart dust, which consists of a microprocessor, sensors, and communication systems and power sources that can form a wireless network in a self-organizing manner. The smart dust can locate each other and transmit information to the observer. The literature has embarked on a research program on WSNs [5], and some companies have started to study the "Development plan for a new type of computing based on satellite sensor networks." Numerous scholars and institutions have researched WSNs. Up to now, the latest research has focused on improving the efficiency of energy usage, focusing on routing algorithms, clustering algorithms, communication protocols, etc. The literature develops an energy-efficient hierarchical data distribution protocol for sensor-to-edge server communication that supports dynamic changes in network topology and has significant advantages in terms of energy consumption in harsh environments, green habitat design, and coverage [6]. The literature proposes a novel WSN routing protocol that applies to the mobility of sensor nodes for reliable and energy-efficient routing. The literature uses butterfly optimization algorithm (BOMA) to select the cluster heads and selects the optimal route based on ant colony optimization (ACO), which greatly extends the green habitat design. Innetwork convergence current research is still in the preexploration stage. The literature analyzes the challenges and opportunities of WSN and wireless mesh network (WMN) convergence and proposes and compares several convergence approaches. The literature proposes a fiber-wireless sensor network (Fi-WSN) gateway design that achieves seamless convergence of passive optical networks (PON) and WSNs [7].

The literature has studied "key technology and coordination control theory of distributed self-control system on sensor networks" as a key research project. The literature has studied the "network foundation theory of wireless sensing," and in 2008, the national major project has also made sensing network technology a key research area. Many research institutions have joined in the research of WSNs. So far, research on WSNs is still ongoing [8]. The literature extends the green habitat design of mobile wireless sensor network (MWSN) by developing a system model and using different algorithms for optimization. The literature proposes fuzzy multihop clustering protocol (FMSFLA) using shuffled frog leaping algorithm (SFLA) which considers energy, distance to the base station, number of neighboring nodes, actual node to base station distance, average routing load, delay [9], and other parameters, which is superior to LEACH, LEACH-EP, LEACH-FL, and other protocols for green habitat design. The literature proposes an improved LEACH algorithm for low-power adaptive set clustering algorithm (LEACH) with random b cluster head selection, no consideration of node-to-base station distance, unreasonable node distribution, and uneven energy consumption. Compared to the original algorithm, green habitat design is improved by $36.4 \%$. There have been many exploratory studies on network fusion in wireless sensor networks. The literature proposes the network fusion of MCN and WSN using mobile terminals in mobile cellular networks (MCN) as both sensor nodes and WSN gateways. By fusing WSN and group-wise 
sensing networks, the literature enables both networks to borrow each other's advantages to achieve more powerful intelligent sensing effects [10].

In wireless sensing harmful substance detection of green habitat environment, the development is also faster; as early as the 1960s and 1970s, developed countries in environmental intelligence and mechanization made plans [11]; the relevant environmental departments first proposed the concept of intelligent environment; developed countries proposed the integration of emerging Internet technology and intelligent environment and synergistic development. With the advantages in technology, the technological innovation related to the wisdom environment is constantly explored; seeking the smallest human input in exchange for the largest and most stable environmental production capacity, electronic integrated circuit technology has advanced by leaps and bounds, and the miniaturization and intelligence of components have changed the development process of human society; along with the emergence of microcomputer technology, people are more optimistic about the development prospects of the wisdom environment, and at the same time, with the microcomputer system. At the same time, with the emergence of microcomputer systems and microsensor chips, the combination of intelligent environment and computer Internet technology has reached a new height [12]. The developed countries represented by the United States and Japan, relying on advanced electronic integrated circuit technology and complete industrial chain, have made great achievements in environmental informationization, applying the concept of informationization to environmental development through microcomputer systems [13]; for example, computer systems plus peripherals can be used to sensors to monitor crop growth, by changing the temperature to regulate the growth cycle of crops; this is suitable for environmental greenhouses and can effectively eliminate the influence of the external environment; in addition to the regulation of temperature and humidity, other important growth factors can also be monitored and regulated [14], such as real-time monitoring of $\mathrm{CO} 2$ concentration in the environment; photosynthesis is the most important process of crop growth, through the computer system plus. The environmental monitoring platform built by computer system and peripheral sensors can monitor the CO2 concentration of the growing environment of crops in real time and then determine whether it is conducive to the growth and development of crops [15] and can be regulated when it is too high or too low, so as to ensure the growth quality and yield of crops with the most suitable concentration [16].

\section{Green Habitat Design Based on Field Wireless Sensing Hazardous Substance Detection}

\subsection{Detection of Hazardous Substances by Field Wireless} Sensing. The most used in the detection of hazardous substances in the field of wireless sensing is ZigBee technology; ZigBee is a short-range transmission, low energy consumption, and low rate of wireless network technology; it is a wireless network communication scheme between RFID (radio frequency identification) and Bluetooth. ZigBee is based on IEEE 802.15.4 standard, which mainly specifies the application layers that can be shared by various manufacturers. The standard mainly defines the physical layer and the network layer. The physical layer is defined as the transport of information at a rate of $250 \mathrm{~kb} / \mathrm{s}$ at $2.4 \mathrm{GHz}$ and at lower rates of $915 \mathrm{Mz}$ and $868 \mathrm{MHz}$, with certain specifications for the physical layer. The specifications for the network layer define specific issues related to the sharing of air channels between different radio signals in the same area during operation [17]. The advantages of wireless sensors are (1) no need to connect, which reduces installation costs; (2) there is no direct electrical connection between each other, and the problem of mutual interference does not exist; and (3) some conditions cannot be connected, and wireless sensors must be used.

ZigBee has been widely used in sensor networks, the Internet of Things, and other fields, mainly because of its very powerful ability in networking. ZigBee network has three network topologies, namely, star topology, tree topology, and mesh topology; users can choose three network structures according to their needs. Each of these three ZigBee network structures has its advantages. The tree topology mainly consists of a coordinator and multiple routers and end nodes. The principle formula is as follows.

$$
T=\frac{1}{n} \sum_{i=1}^{n} X_{i}+\sum_{i=1}^{n} X_{i} Y_{i}
$$

This formula puts forward the basic content of wireless sensors, how to make the signal better, and can provide a better creative environment for creators. The coordinator is capable of connecting multiple routers and terminals, and the coordinator is capable of connecting multiple routers and terminals from the node's router. Thus, the entire network will contain several layers, and the details of the structure are shown in Figure 1.

The main components of network topology are a coordinator, several routers, and terminals. It has some similarities with the tree topology. However, the routing paths are more flexible, and the routing nodes can be connected and pass information to each other according to their actual needs. The principle of the formula is as follows.

$$
A=\sum_{i=1}^{n} X_{i}^{2}+\frac{1}{n}\left(\frac{x-\mu}{\sigma}\right) .
$$

This flexible routing allows for more efficient delivery of data, and this routing has more robustness, where one path is blocked in the network; data can have other paths for message delivery. The mechanism of mesh topology is shown in Figure 2.

Generally, when implementing a mesh network, the corresponding routing protocols are developed at the network layer, which enables the nodes to use the optimal path for data transmission, increases the efficiency of network data transmission, and to some extent reduces the energy 


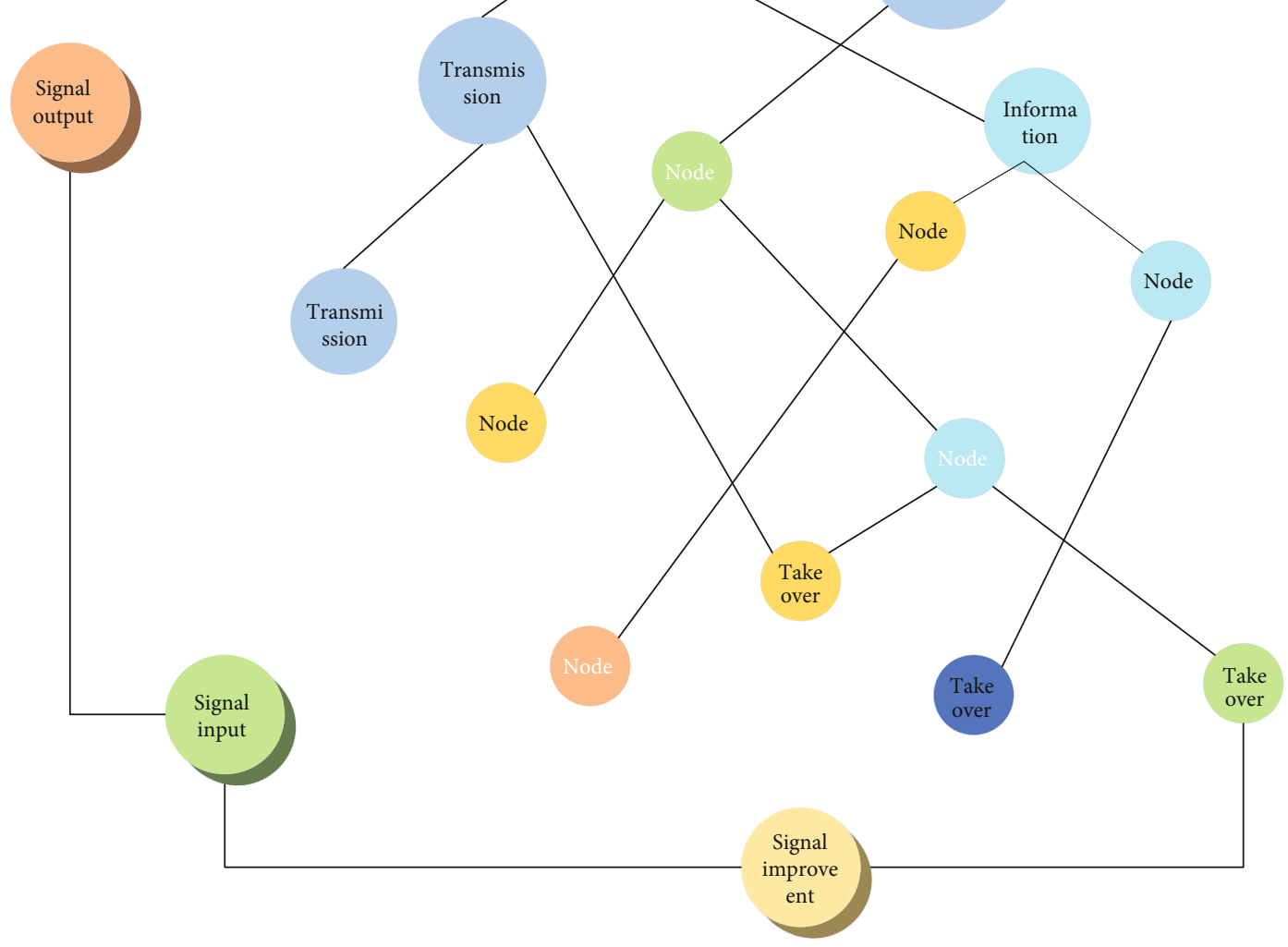

Figure 1: Tree topology diagram.

consumption of the network. All the abovementioned features are implemented by the network layer, and the application layer does not need to perform any involvement. The mesh structure is very powerful and can deliver information in the form of "multi-level hops," and it can form a network with higher complexity based on this structure; in addition, it can also achieve self-organization and self-healing [18].

NB-IoT (narrow band Internet of Things), a technology that has not been in people's view for a long time, is based on cellular networks in the field of IoT. NB-IoT is currently evolving from traditional cellular IoT technology to the more advanced LPWAN, an improvement on existing cellular communications to accommodate low-power wide-area interconnections, driven by mobile operators and the equipment vendors behind them. For power wide-area network, NB-IoT is aimed at addressing four major pain points of current cellular networks for LPWA applications.

\section{(1) Broad coverage}

In other words, it is 100 times more capable of receiving signals than the mobile network, and the principle formula is as follows.

$$
T=\frac{\Delta y}{\Delta x} \cdot \frac{\delta y}{\delta x} \cdot \frac{\partial^{2} \Omega}{\partial u^{2}}
$$

To a great extent, it strengthens the network coverage power. So full coverage can be achieved through it for areas that cannot be effectively covered by the current mobile network, e.g., suburbs, large factories, high-rise, elevators, and floor underground garages.

\section{(2) Large connection}

NB-IoT technology increases the number of accesses by 50 to 100 times over existing cellular wireless technologies. It can accommodate up to 50,000 user equipment (UE) connections to the core network in an area. The principle of the formula is as follows.

$$
T=\sum_{i=1}^{n} X_{i} Y_{i}+\left(\frac{x-\mu}{\sigma}\right)
$$

\section{(3) Low power consumption}

The key factor in achieving this feature is the effective streamlining of some signaling that is not necessary or its application to longer paging tasks. The reduction in power consumption achieved by some of these means, such as some terminal modules that require a long life cycle, is 


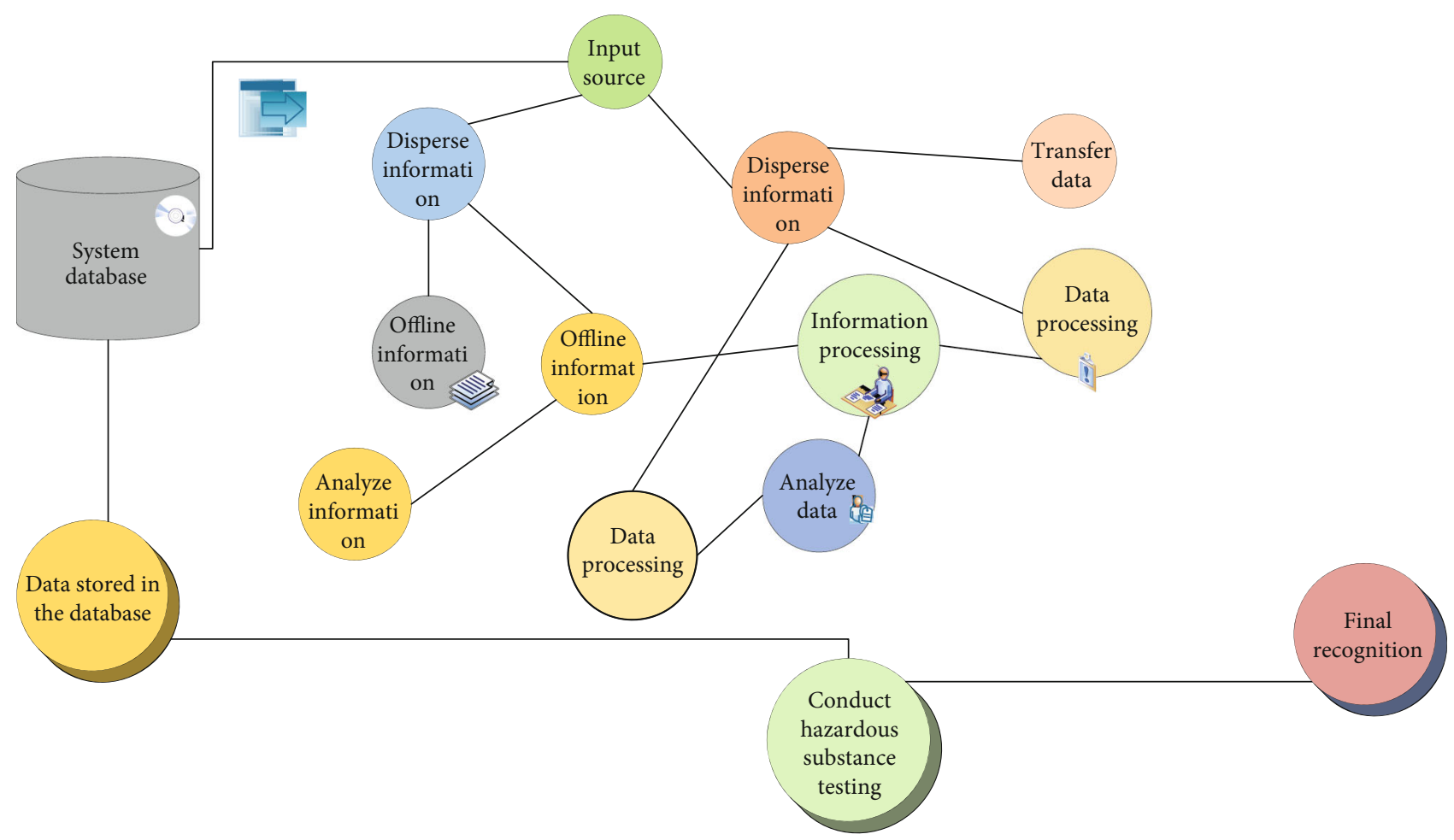

Figure 2: Mesh topology diagram.

estimated to reach a standby time of ten years. The formula is as follows.

$$
T=\arcsin \theta \cdot\left(\frac{\pi}{2}-\theta\right)
$$

(4) Low cost

NB-IoT achieves low cost primarily by limiting the data rate, energy usage, and bandwidth of end devices, allowing for effective simplification of the endpoint as a whole. At present, the expected price of a single module is not more than $\$ 5$ and may still fall. From the above, NB-IoT has high applicability to many fields. NB-IoT technology works in the authorized spectrum and focuses on low power consumption, wider coverage requirements, etc. proposed by IoT. It is suitable for IoT applications that do not require high information transmission rate and need ultralow device cost requirements and low device power consumption [19].

Green environmental design refers to incorporating environmental factors into the design to help determine the direction of design decisions. Green environmental design requires consideration of environmental factors in all stages of product development, reducing the impact on the environment from the entire life cycle of the product and ultimately leading to a more sustainable production and consumption system. It focuses on the relationship between the landscape spatial pattern and the spatial pro- cess. The landscape spatial pattern is composed of patches, substrates, corridors, borders, and other elements.

The sensor node in the field is a very tiny system that is mainly responsible for collecting information and carrying out related processing. It mainly contains modules such as sensors, information processing, and wireless communication. The various modules of the sensor node meet different task requirements. The sensor module is mainly used to collect and convert various data in the target area; the information processing module is mainly responsible for the management of the received information data, such as the entire sensor node storage and its collection of various data or data received by other nodes and sent; wireless communication module is mainly to complete the exchange of data between the various sensor nodes; the main task of the power supply module is to provide power for the normal operation of the entire wireless sensor network. The main task of the power supply module is to provide powerful support for the normal operation of the entire wireless sensor network; in the entire field, the WSN system can continue to run; then, you can detect harmful substances; the detection process is shown in Figure 3.

Wireless sensors can play an important role in the detection and prevention of meteorological natural disasters. In the research of various atmospheric phenomena, weather disaster prevention monitoring, flood, and fire monitoring, wireless sensors can all play a role. Wireless sensors can play an important role in the detection of the ecological environment. In addition, China as a developing country, in the past, achieves rapid economic growth but failed to pay sufficient attention to the protection of resources, ecology, etc., 


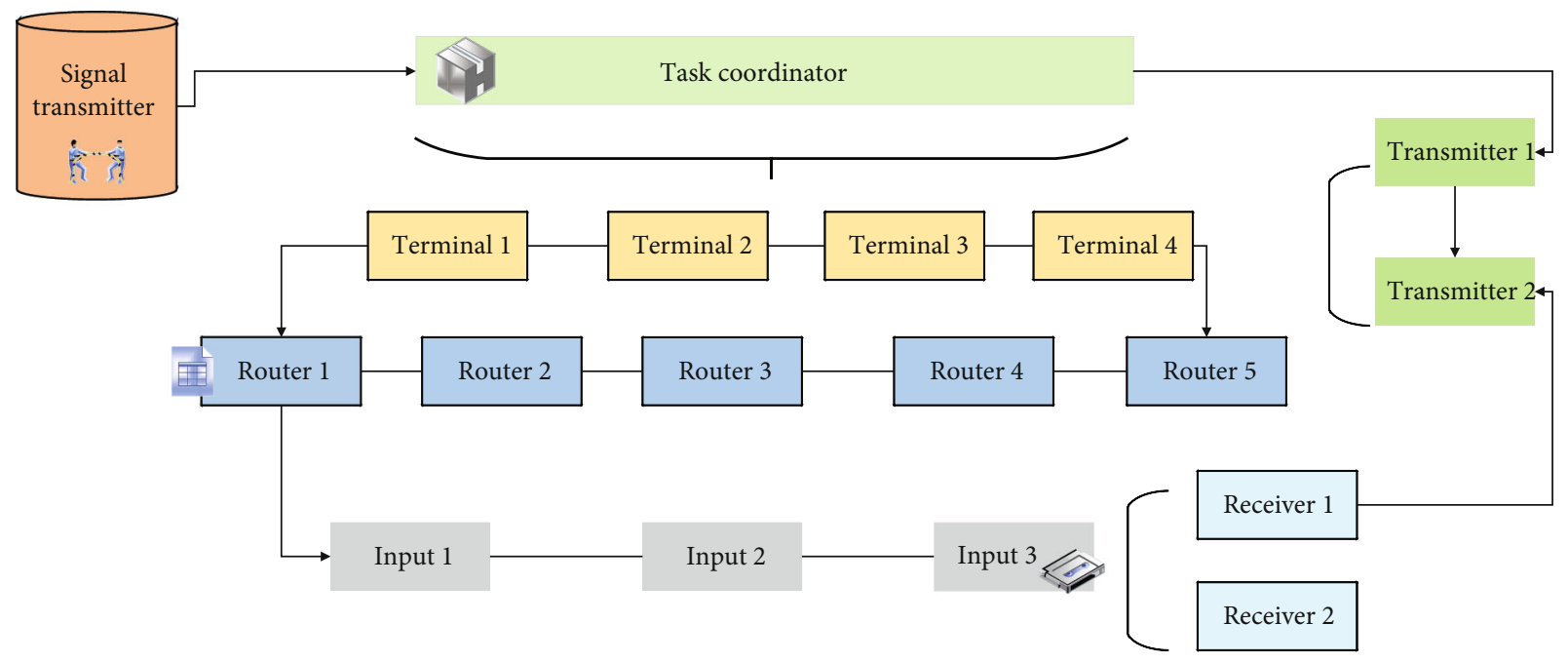

FIGURE 3: WSN hazardous substance detection process.

resulting in the current development of significant uneven and inadequate characteristics; the overall quality level is low, the ecological constraints on development is increasingly prominent, and prosperous ecological culture is the need of the times, to promote the sustainable improvement of ecological, and environmental quality is an important task. Wireless sensors also play a major role in the construction of intelligent ecological and environmental management systems [20]. In addition to its role in the construction of environmental ecology, it can also make its contribution in the field of the production of harmful substances. For example, the use of wireless sensors in the production of hazardous substances can play its role in accurately monitoring all kinds of environmental changes that may occur in the production of hazardous substances, thus largely avoiding unexpected factors that cause crop freezing, pests, and other difficult problems. This is also of great significance to the stable and prosperous development of the market economy.

3.2. Green Habitat Design. For the design of green habitat environment based on field wireless sensing hazardous substance detection, the design area is a village, and its design content mainly includes modern hazardous substance area, courtyard greenhouse area, hazardous substance display test area, ecological recycling facilities planning content mainly includes modern hazardous substance area, courtyard greenhouse area, hazardous substance display test area, ecological recycling facilities, and modern hazardous substance area design; the field is relatively flat and is the only concentrated farmland in the village. The rainfall is abundant during the rainy season, and the plan adds interception and storage facilities so that there is water all year round after completion, which is more suitable for the development of hazardous substances. The ecological greenhouse is introduced based on the original one, so that the production and experience of harmful substances can be carried out all year round, creating a four-season paradise for harmful substances. The area covers an area of $160 \mathrm{mu}$, which is renewed to grow food and vegetables and is the main source of living resources for the villagers and the migrant population. The college is a living space, mainly in the courtyard greenhouse. Here, we can increase the living space of foreign mobile people and planting space; people can live in the natural oxygen bar and live and experience farming activities, providing a completely different residential life model from the city. The design process is shown in Figure 4.

In terms of ecology, ecological recycling facility is a system of facilities including biogas stations and sewage ecological sedimentation ponds; this is the core of the entire green village cycle; the healthy operation of the ecological recycling center is the key to ensure the recycling of resources in the village, so the equipment requirements, scale requirements, and location selection are critical. Ensure that the treatment capacity and gas production capacity meet the waste discharge and gas demand in the village. The main purpose is to anaerobically ferment the manure and organic waste produced in the production and living system and transform them into biogas gas resources, while the digestate and methane are used for fertilization of farmland. A closedchain cycle model of straw-cultivation-manure-biogasdigestion liquid used in farmland fertilization is formed [21]. Green habitat also includes green light, and the development of the habitat light environment has become more diverse than the original single lighting function, and the use and selection of light have become more complex. The living environment not only accommodates chandeliers, desk lamps, and lighting street lamps to meet people's daily life functions but also develops decorative lighting projection lamps, pipe lamps and advertising lighting neon lights, optical fibers, etc. It can be seen that the reason for the complex use of lighting in the light environment is mainly due to the variety of uses of lamps and light sources and the complexity of the types of light sources [22].

\section{Experimental Results and Analysis}

4.1. Experimental Results. After the mobile sensor nodes have completed traversing the area, repairing coverage voids, 


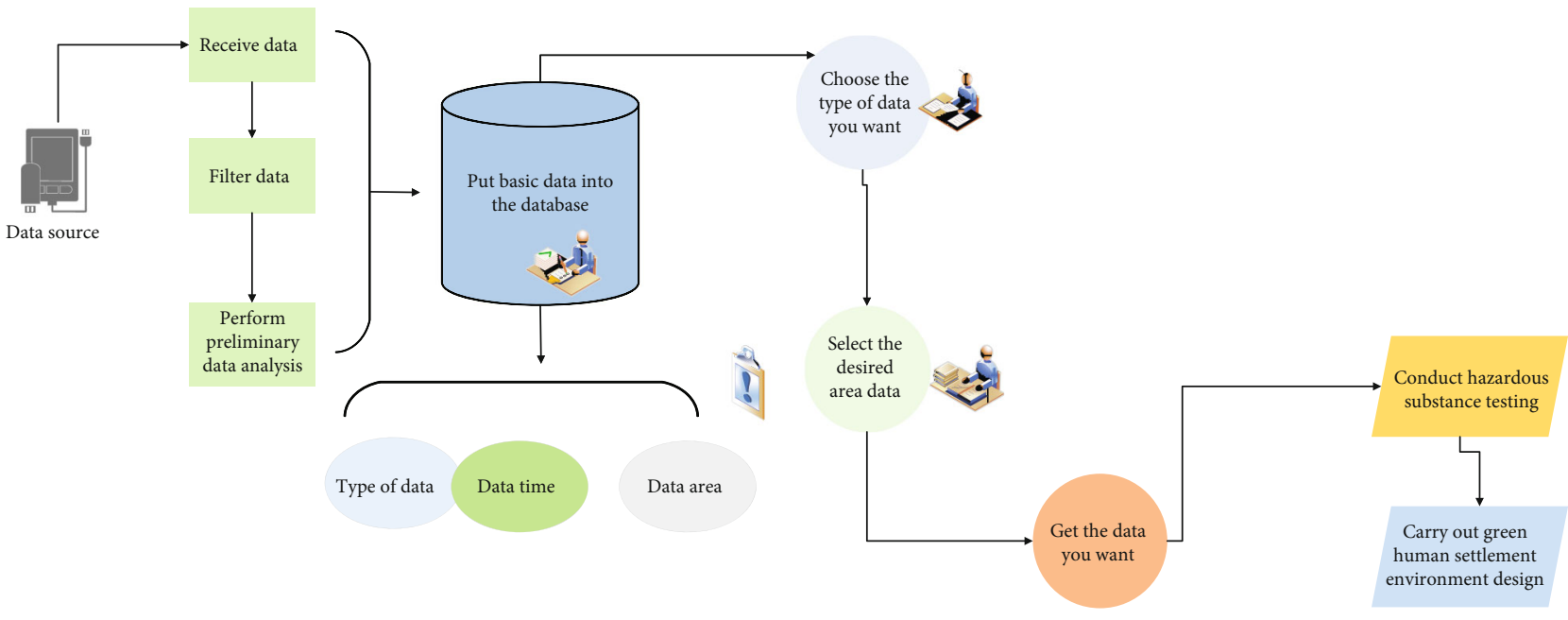

FIgURE 4: Green habitat design process.

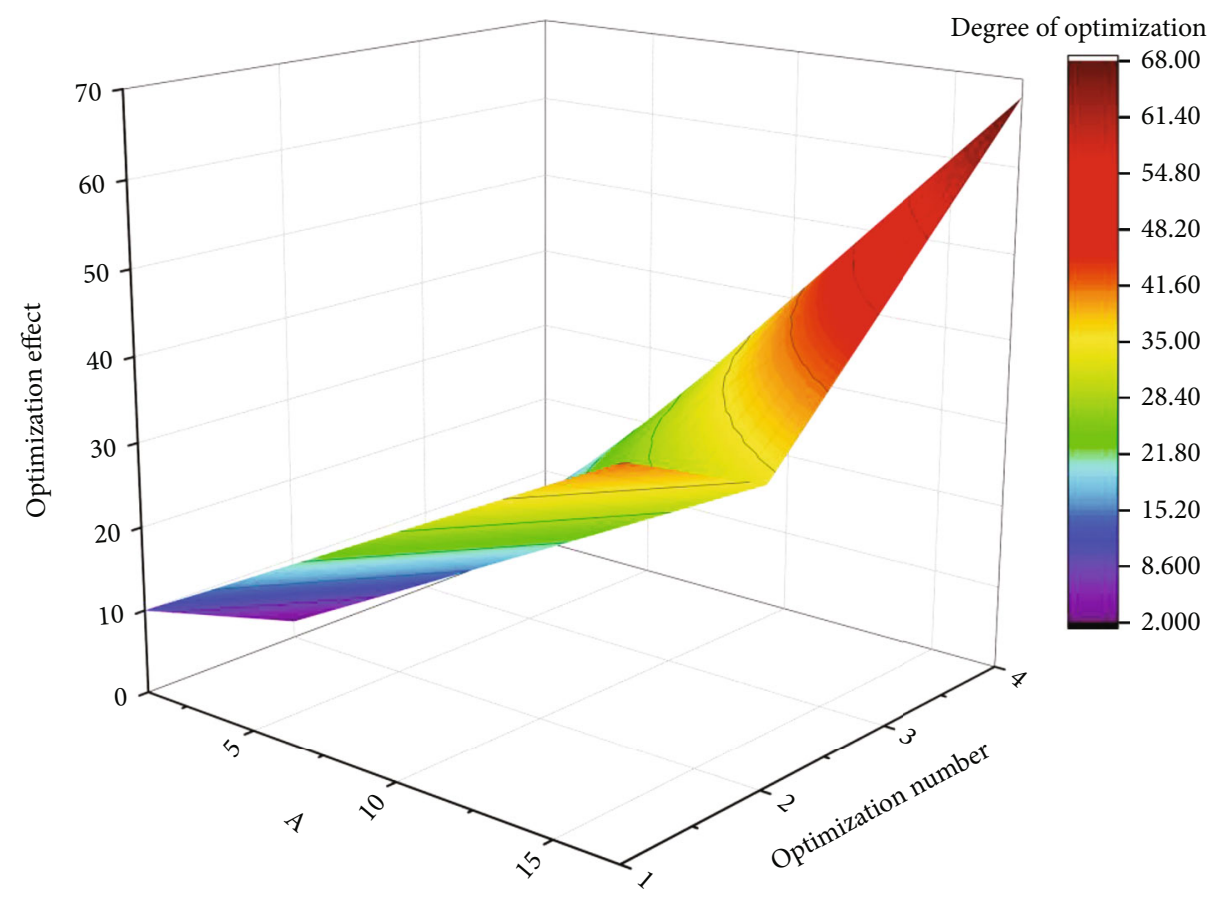

FIgURE 5: Comparison of efficiency after optimization.

and collecting data, establishing an efficient data forwarding mechanism can effectively reduce the overall energy loss of the network, extend the working cycle of the network, detect harmful substances, and design a green habitat environment, and the following efficiency comparisons are obtained after comparing the details through optimization, as shown in Figure 5.

The method of evaluating the green habitat design is often evaluated by the number of nodes surviving, which is defined in this paper as the number of rounds elapsed from the beginning of the simulation to the end when the number of nodes surviving is less than $10 \%$ of the total number of nodes. In this paper, 96 sensing nodes at the same location are used to simulate and test the node survival of AODV protocol, AODV-based wireless sensor network routing protocol, LEACH-based single-hop clustered routing protocol, and secondary mesh wireless sensor network clustered routing protocol for each protocol. The first node failure occurs later in AODV-based wireless sensor network routing protocol compared to AODV protocol because AODV protocol always uses the route with a minimum number of hops and does not consider the energy consumption of the transit node; thus, the energy of the node on the route with a minimum number of hops will be depleted quickly, and the node failure remains the same after changing the route and considers the remaining energy of the node when selecting the route and thus can start working for a longer period without node failure. Since both protocols have more similar 


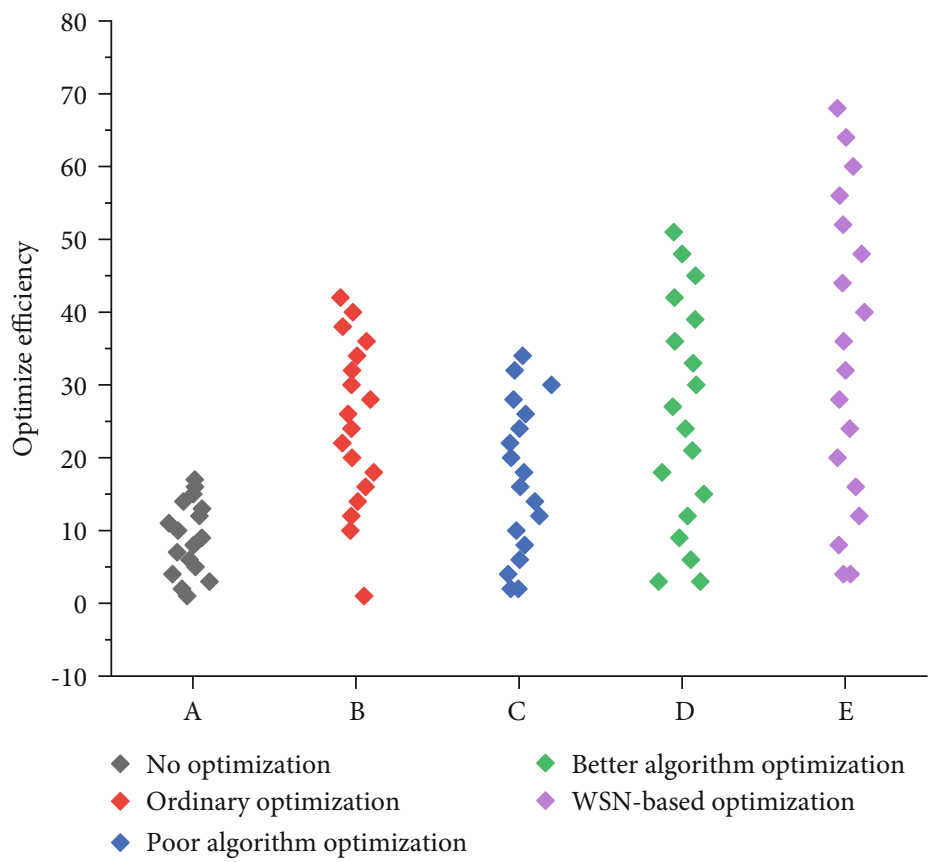

Figure 6: Graph of feasibility results.

algorithms for route discovery, there is not much difference in green habitat design, and AODV-based wireless sensor network routing protocol performs slightly better than the AODV protocol for green habitat design. The reason for the difference in green habitat design is mainly due to the elimination of Hello broadcast and the shorter message length of the AODV-based wireless sensor network routing protocol, thus reducing the overall energy consumption of the network and extending the sustainability of the green habitat design. The final feasibility results are shown in Figure 6.

4.2. Experimental Analysis. Both the clustered routing protocols outperform the planar routing protocols because the clustered routing protocols reduce the routing messages as well as the length of data messages across the network through data fusion. One of the secondary mesh wireless sensing network cluster routing protocol cluster heads is connected to the normal nodes through multihop connections with more nodes in the cluster, further reducing the energy used to transmit the sensing information within the cluster. The simulation test of routing energy overhead is to count the number of messages sent by the entire network when a node establishes a route to a target node and the energy consumption. In this chapter, sensing node 96 in the upper left corner is chosen as the source node, and sensing node 64 in the lower right region is the target node. The simulation process is to broadcast the route request from sensing node 96 until the route is established, and the simulation results of each protocol are shown in Figure 7. Although the AODV protocol and the AODV-based wireless sensor network routing protocol send the same number of messages during the simulation, the latter has a shorter message length and has an advantage in the overall energy consumption of the network. The LEACH-based singlehop cluster routing protocol still has a higher total number of sends due to more cluster head nodes and more routing messages built by the cluster head, which carries a larger network load. Methods for detecting harmful substances include field-based wireless sensor network (WSN), X-ray fluorescence spectroscopy (X-ray), Fourier infrared spectroscopy (FIS), and cold atomic absorption spectroscopy (CAS). The secondary mesh wireless sensor network cluster routing protocol generates fewer cluster heads which effectively controls the amount of routing messages in the network and also reduces the consumption in the process of building routes; the simulation results for each protocol are shown in Figure 7.

The control chip of the sensing node is ESP32, which has a high-performance LX6 dual-core processor with a high level of low-power performance. This chip has a complete software development kit and supports Arduino development, which can greatly improve development efficiency. The energy management chip of the sensing node is the bq25570, which collects the power generated by the solar cell and stores it in the battery through an integrated boost converter and also provides a set voltage for the system through an integrated high-efficiency, micropower buck converter. The node can collect information from three environments, temperature, vibration, and sound, using a temperature digital sensor DS18b20, a three-axis acceleration sensor ADXL345 and a digital MEMS sensor INMP441, respectively. These sensors are small and easy to integrate into the system. The output is also digital, eliminating the need for additional noise reduction and analog-to-digital conversion circuitry. The network fusion method of the hardware testbed is a personal domain network device transmitting information through a wireless sensor network. The RF chip 


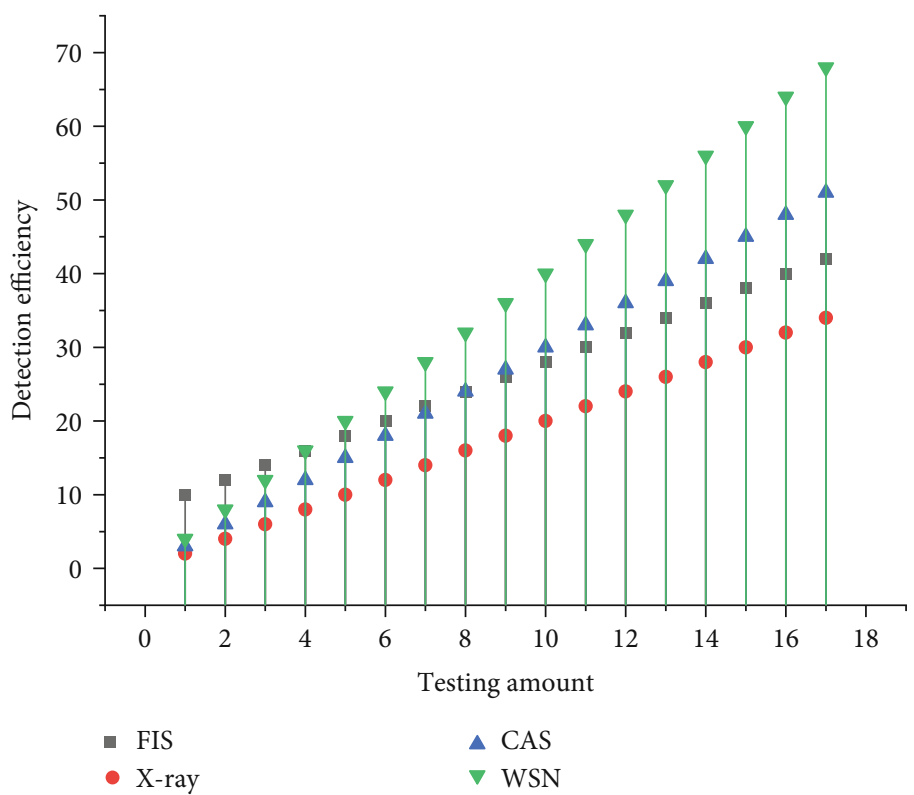

FIGURE 7: Simulation results for each protocol.

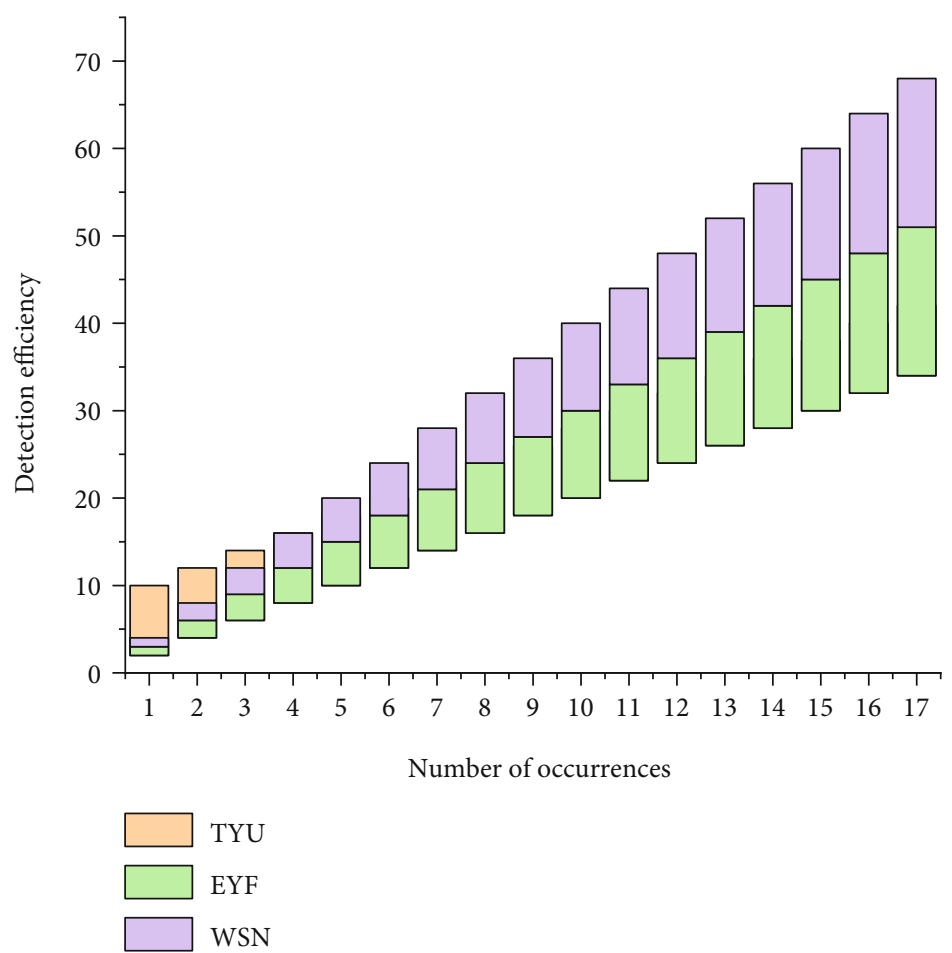

FIgURE 8: Efficiency diagram of the design role of human settlements.

used in the personal area network is nRF24L01, which operates in the frequency range of $2.400 \mathrm{GHz} 2.525 \mathrm{GHz}$, with a transmission rate of up to $2 \mathrm{Mbps}$ and low power consumption, and is connected to the control chip using the SPI bus. The CC1101 is selected for the wireless sensor network; the chip's operating frequency range is $387.0 \mathrm{MHz} 464.0 \mathrm{MHz}$, with low signal loss and long transmission distance compared to the high-frequency range. The CC1101 does not come with a communication protocol, so it is suitable for implementing the designed routing protocol based on this chip. The efficiency diagram of its design role for habitat is shown in Figure 8.

The software platform of the intelligent hazardous material monitoring system mainly includes the Raspberry Pi intelligent monitoring interface and the main system program design; we use Python and PyQt5 to develop the above software platform programs. Python language has opensource third-party libraries and advanced performance of 
object-oriented programming, which can greatly shorten the development cycle of the intelligent monitoring platform. The Python language has open-source third-party libraries and advanced object-oriented programming capabilities, which can greatly reduce the development cycle of intelligent monitoring platform, and Python programs have strong compatibility and can be programmed and developed in a variety of operating systems; running Python programs in the microembedded platform does not require too much programmatic basis, only the relevant compiler loaded in the microembedded platform. The Raspberry Pi 3B+ system used in this system comes with a Python compiler, so we only need to use the Python language on the PC side of the smart monitoring software platform development and then through the aforementioned FileZilla software to transfer .py source file to Raspberry Pi 3B+, through the Python compiler; we can open our intelligent monitoring platform on Raspberry Pi 3B+, which can be used to detect harmful substances through a wireless sensor-based system, so that we can better design and implement a green habitat environment, and the final result is satisfactory.

\section{Conclusion}

In this paper, in view of the single data collection, poor compatibility of software platform, and high cost of construction and maintenance of traditional hazardous substance monitoring system, the intelligent hazardous substance monitoring system is designed by using wireless sensor network technology, microembedded technology, and image acquisition technology, and this paper does the analysis and combing of the current situation of domestic and international development of intelligent hazardous substance and hazardous substance informatization and intelligence and summarizes the current traditional based on the functionality and coverage of the system data acquisition network; the ZigBee network protocol is chosen for the construction of wireless sensor network and data transmission, and the commonly used ZigBee chips are introduced and compared, and the CC2530 chip with low power consumption and easy networking is selected as the hardware carrier of the wireless sensor network; the types of sensors and deployment methods are determined according to the data to be collected; the fixed nodes are identified as the wireless sensor network collection units, and the hardware and software design schemes of the fixed nodes are given. The front-end fixed node can collect temperature, humidity, and $\mathrm{pH}$ data stably; the mobile quadruped node can switch walking posture in the farmland, and the automatic photo module can collect image information in real-time. The algorithm should improve the recognition efficiency in the future, and it will be more helpful to the design of a green environment.

\section{Data Availability}

The data used to support the findings of this study are included within the article.

\section{Conflicts of Interest}

No competing interests exist concerning this study.

\section{References}

[1] S. Olenik, H. S. Lee, and F. Güder, "The future of near-field communication-based wireless sensing," Nature Reviews Materials, vol. 6, no. 4, pp. 286-288, 2021.

[2] A. Uchiyama, S. Saruwatari, T. Maekawa, K. Ohara, and T. Higashino, "Context recognition by wireless sensing: a comprehensive survey," Journal of Information Processing, vol. 29, pp. 46-57, 2021.

[3] A. R. Al-Qawasmi and A. Abokhalil, "Smart energy management system using wireless sensing and actuator network," Journal of Engineering and Applied Sciences, vol. 8, no. 1, pp. 9-19, 2021.

[4] S. J. Hsiao and W. T. Sung, "Employing blockchain technology to strengthen security of wireless sensor networks," IEEE Access, vol. 9, pp. 72326-72341, 2021.

[5] S. Kato, T. Fukushima, T. Murakami et al., "CSI2Image: image reconstruction from channel state information using generative adversarial networks," IEEE Access, vol. 9, pp. 4715447168, 2021.

[6] H. Kasban, S. Nassar, and M. A. M. El-Bendary, "Medical images transmission over wireless multimedia sensor networks with high data rate," Analog Integrated Circuits and Signal Processing, vol. 108, no. 1, pp. 125-140, 2021.

[7] C. T. Beranek, J. Clulow, and M. Mahony, "Wetland restoration for the threatened green and golden bell frog (Litoria aurea): development of a breeding habitat designed to passively manage chytrid-induced amphibian disease and exotic fish," Natural Areas Journal, vol. 40, no. 4, pp. 362-374, 2020.

[8] Y. Xie, S. Yao, Y. Deng, L. Jia, Y. Li, and Q. Gao, "Impact of the 'Grain for Green'project on the spatial and temporal pattern of habitat quality in Yan'an City, China," Chinese Journal of EcoAgriculture, vol. 28, no. 4, pp. 575-586, 2020.

[9] A. Prasad, J. Pedlar, M. Peters et al., "Combining US and Canadian forest inventories to assess habitat suitability and migration potential of 25 tree species under climate change," Diversity and Distributions, vol. 26, no. 9, pp. 1142-1159, 2020.

[10] J. G. Mills, A. Bissett, N. J. C. Gellie et al., "Revegetation of urban green space rewilds soil microbiotas with implications for human health and urban design," Restoration Ecology, vol. 28, no. S4, pp. S322-S334, 2020.

[11] K. Metcalfe, N. Bréheret, G. Bal et al., "Tracking foraging green turtles in the Republic of the Congo: insights into spatial ecology from a data poor region," Oryx, vol. 54, no. 3, pp. 299-306, 2020.

[12] S. Piovano, G. E. Lemons, A. Ciriyawa, A. Batibasaga, and J. A. Seminoff, "Diet and recruitment of green turtles in Fiji, South Pacific, inferred from in-water capture and stable isotope analysis," Marine Ecology Progress Series, vol. 640, pp. 201-213, 2020.

[13] T. Eguchi, J. Bredvik, S. Graham, R. LeRoux, B. Saunders, and J. A. Seminoff, "Effects of a power plant closure on home ranges of green turtles in an urban foraging area," Endangered Species Research, vol. 41, pp. 265-277, 2020.

[14] N. Xiu, M. Ignatieva, C. K. van den Bosch, and S. Zhang, "Applying a socio-ecological green network framework to Xi'an City, China," Landscape and Ecological Engineering, vol. 16, no. 2, pp. 135-150, 2020. 
[15] B. F. da Silva, J. C. Pena, A. B. Viana-Junior, M. Vergne, and M. A. Pizo, "Noise and tree species richness modulate the bird community inhabiting small public urban green spaces of a Neotropical city," Urban Ecosystem, vol. 24, no. 1, pp. 71-81, 2021.

[16] R. W. F. Cameron, P. Brindley, M. Mears et al., "Where the wild things are! Do urban green spaces with greater avian biodiversity promote more positive emotions in humans?," Urban Ecosystem, vol. 23, no. 2, pp. 301-317, 2020.

[17] K. E. Plummer, S. Gillings, and G. M. Siriwardena, "Evaluating the potential for bird-habitat models to support biodiversityfriendly urban planning," Journal of Applied Ecology, vol. 57, no. 10, pp. 1902-1914, 2020.

[18] S. Saura, "The habitat amount hypothesis implies negative effects of habitat fragmentation on species richness," Journal of Biogeography, vol. 48, no. 1, pp. 11-22, 2021.

[19] A. J. Rodríguez, J. A. Ricardo, E. G. Alfonso, R. B. Ávila, C. C. Guerra, and R. C. Peña, "Prevalence of fibropapilloma in Chelonia mydas (Testudines, Cheloniidae) juveniles and environmental quality of their habitat at north of Villa Clara, Cuba/Prevalencia de fibropapilomas en juveniles de Chelonia mydas (Testudines, Cheloniidae) y calidad ambiental de su hábitat en el norte de Villa Clara, Cuba," Revista de Investigaciones Marinas, vol. 41, no. 1, pp. 91-105, 2021.

[20] E. Andersson, D. Haase, S. Scheuer, and T. Wellmann, "Neighbourhood character affects the spatial extent and magnitude of the functional footprint of urban green infrastructure," Landscape Ecology, vol. 35, no. 7, pp. 1605-1618, 2020.

[21] J. I. Watling, V. Arroyo-Rodríguez, M. Pfeifer et al., “Support for the habitat amount hypothesis from a global synthesis of species density studies," Ecology Letters, vol. 23, no. 4, pp. $674-681,2020$.

[22] A. Prazukin, N. Shadrin, D. Balycheva, Y. Firsov, R. Lee, and E. Anufriieva, "Cladophora spp.(Chlorophyta) modulate environment and create a habitat for microalgae in hypersaline waters," European Journal of Phycology, vol. 56, no. 3, pp. 231-243, 2021. 